\title{
Review
}

\section{Do Psychosocial Interventions Improve Quality of Life and Wellbeing in Adults with Neuromuscular Disorders? A Systematic Review and Narrative Synthesis}

\author{
Elaine Walklet ${ }^{\mathrm{a}, \mathrm{b}, *}$, Kate Muse $^{\mathrm{b}}$, Jane Meyrick ${ }^{\mathrm{a}}$ and Tim Moss ${ }^{\mathrm{a}}$ \\ ${ }^{\mathrm{a}}$ Health and Social Sciences Department, University of the West of England, Bristol, UK \\ ${ }^{\mathrm{b}}$ Psychology Department, University of Worcester, Worcester, UK
}

\begin{abstract}
Quality of life and well-being are frequently restricted in adults with neuromuscular disorders. As such, identification of appropriate interventions is imperative. The objective of this paper was to systematically review and critically appraise quantitative studies (RCTs, controlled trials and cohort studies) of psychosocial interventions designed to improve quality of life and well-being in adults with neuromuscular disorders. A systematic review of the published and unpublished literature was conducted. Studies meeting inclusion criteria were appraised using a validated quality assessment tool and results presented in a narrative synthesis. Out of 3,136 studies identified, ten studies met criteria for inclusion within the review. Included studies comprised a range of interventions including: cognitive behavioural therapy, dignity therapy, hypnosis, expressive disclosure, gratitude lists, group psychoeducation and psychologically informed rehabilitation. Five of the interventions were for patients with Amyotrophic Lateral Sclerosis (ALS). The remainder were for patients with post-polio syndrome, muscular dystrophies and mixed disorders, such as Charcot-Marie-Tooth disease, myasthenia gravis and myotonic dystrophy. Across varied interventions and neuromuscular disorders, seven studies reported a short-term beneficial effect of intervention on quality of life and well-being. Whilst such findings are encouraging, widespread issues with the methodological quality of these studies significantly compromised the results. There is no strong evidence that psychosocial interventions improve quality of life and well-being in adults with neuromuscular disorders, due to a paucity of high quality research in this field. Multi-site, randomised controlled trials with active controls, standardised outcome measurement and longer term follow-ups are urgently required.
\end{abstract}

Keywords: Neuromuscular diseases, adult, quality of life, psychology, review

\section{INTRODUCTION}

Neuromuscular disorders have a high symptom burden and are frequently associated with many

\footnotetext{
${ }^{*}$ Correspondence to: Elaine Walklet, Department of Psychology, University of Worcester, Henwick Grove, WR2 6AJ, UK. Tel.: +44 01905 542420; E-mail: e.walklet@worc.ac.uk.
}

adverse psychosocial outcomes, particularly reduced quality of life and well-being [1,2]. The World Health Organisation (WHO) defines quality of life as the way in which an individual perceives their individual position in life with respect to their goals and standards and suggests this broad construct can be affected by the individual's physical health, psychological health, independence, and relationships 
with others and the environment [3]. Health-related quality of life (HRQoL) is one aspect of this multifaceted concept and refers to the physical, social and psychological domains of health, influenced by an individual's beliefs, expectations and experiences [4]. Despite clinical variation between slowly progressive (e.g. Facioscapulohumeral Muscular Dystrophy) and rapidly progressive (e.g. Amyotrophic Lateral Sclerosis) neuromuscular conditions, perceptions of self-reported quality of life and HRQoL are comparably low in individuals with different types of neuromuscular disorders [5, 6].

Recently, researchers have noted that quality of life assessments typically focus on deficits in functioning and have argued that an absence of deficits is not synonymous with positive functioning. Consequently, it has been suggested that well-being, an asset approach to functioning assessing positive psychological variables, should also be considered as an important outcome in chronic illness [7, 8]. Indeed recent definitions of health now emphasise positive psychological factors, namely the ability to adapt and self-manage [9]. Arguably a difficult construct to define, one key conceptualisation of well-being, subjective well-being, has been described as 'an umbrella term for different valuations that people make regarding their lives, the events happening to them, their bodies and minds, and the circumstances in which they live' [10]. In contrast, psychological well-being encompasses concepts such as personal growth, mastery, resilience and acceptance [11]. Limited available research suggests that well-being may be important in neuromuscular disorders. For example, research has found adults with amyotrophic lateral sclerosis and high well-being have a risk of mortality seven times lower than those experiencing distress, irrespective of disease severity and length of illness [12].

Given the potential importance of both quality of life and wellbeing in the experience of neuromuscular disease, it is essential that effective interventions are identified. Indeed both patients with neuromuscular disorders and researchers have argued this should be a research priority $[13,14]$. Understanding factors which affect quality of life and well-being is important for the design and delivery of effective interventions. Critically, research has consistently found that psychosocial factors are better predictors of quality of life than physical impairment in adults with neuromuscular disorders [15-17]. Such findings indicate that interventions targeting psychosocial factors could therefore improve quality of life and well-being in this group.
Psychosocial interventions are interventions which target psychological and/or social factors, as opposed to biological factors. These include, for example, all psychological therapies (e.g. cognitive behavioural therapy), psychoeducation and peer support. Such interventions can be delivered in individual and group formats [18]. Systematic reviews and metaanalyses indicate these interventions can improve quality of life and well-being in adults with long-term conditions such as cancer and diabetes [19-21].

To date, no systematic review has considered the effectiveness of psychosocial interventions for improving quality of life and well-being across neuromuscular disorders. This is problematic as the effectiveness of these interventions and methodological quality of the research undertaken to assess effectiveness is largely unknown. Thus far, one published systematic review has assessed psychotherapy and pharmacotherapy interventions for improving well-being and reducing distress in ALS. Based on the results of four included studies, the authors concluded that there was insufficient evidence to make recommendations for psychotherapy [22]. It should be noted, however, that the focus of this review on one aspect of psychosocial interventions (psychotherapy) and a single neuromuscular condition limits the wider applicability of these findings. The importance of reviewing evidence across neuromuscular disorders for non-pharmacological interventions has previously been highlighted but, so far, has been limited to exercise and physical therapy interventions [23]. A systematic review on the effectiveness of psychosocial interventions for improving quality of life and well-being in adults with neuromuscular conditions is now required.

Hence, this review sought to address gaps in the evidence and investigate the effectiveness of psychosocial interventions across neuromuscular disorders, with a view to making recommendations about effective interventions and developments for future research. The aim was to systematically review the literature on the impact of psychosocial interventions on quality of life and wellbeing in adults with neuromuscular disorders.

\section{METHOD}

\section{Search strategy}

A systematic search of published and unpublished literature was conducted between June and 
October 2015. This included the following online databases: PsycInfo (1909-present), PsycArticles (1909-present), Medline (1964-present), CINAHL (1984-present), AMED (1988-present), Web of Science (1900 - present), and the Cochrane Register of Controlled Clinical Trials (CENTRAL). Databases were searched on 9th June 2015 using a Boolean search devised in PsycInfo and adapted to other databases. Due to the small number of studies identified in initial scoping searches, a broad set of free-text search terms were devised around the key concepts of neuromuscular disorders, psychosocial interventions and quality of life/well-being (See Supplementary Material for full search strategy).

A similar search strategy was employed to search COS Conference Papers Index and Open Grey. Hand searching of the Cochrane Neuromuscular Disease Group (all publications) was also conducted. To broaden the search, identified experts in the field and neuromuscular disease charities were also contacted. Citation tracking (using Google Scholar) and hand searching of reference lists were carried out for all included studies.

\section{Inclusion/Exclusion criteria}

To be included in the current review, identified studies had to meet the following criteria:

1. Study design either randomised controlled trial (RCT), controlled trial or cohort study.

2. Participants all adults (18+) with a diagnosis of a neuromuscular disorder. Neuromuscular disorders were defined as disorders of the motor unit, with common examples including motor neuron disorders (e.g. Amyotrophic Lateral Sclerosis [ALS]), nerve junction disorders (e.g. Myasthenia Gravis), muscle disorders (e.g. Duchenne Muscular Dystrophy) and peripheral nerve disorders (e.g. Charcot-Marie-Tooth disease) [24]. In accordance with a previous systematic review, studies of patients with neuromuscular symptoms not attributed to a specific neuromuscular disorder, and studies of patients with diabetic neuropathy, entrapment neuropathy and radiculopathies were not included [23]. Where studies contained mixed samples, $75 \%$ NMD diagnoses was deemed acceptable.

3. Participants received a psychosocial intervention. Psychosocial interventions were defined as interventions targeting psychological and social factors, as opposed to biological or medical.
Common examples include all psychotherapies (such as cognitive behavioural therapy [CBT]), psychoeducation, peer support and expressive disclosure). In accordance with previous reviews, multi-faceted interventions including a biological component but with a clear psychosocial emphasis were included [18].

4. Quality of life and/or well-being of patients assessed as quantitative outcome measure(s). Additional patient specific outcomes (e.g. fatigue, coping etc.) and outcomes for other participant groups (e.g. caregivers) were beyond the scope of this review and, as such, not included.

5. Publication in English language.

\section{Study selection}

To determine eligibility of identified studies, primary screening of titles and abstracts was conducted independently by two of the authors (EW \& KM). Articles that seemed to fulfil the inclusion criteria were subsequently obtained in full-text and subject to independent secondary screening by both authors. To facilitate secondary screening, a data extraction form was developed and used to assess eligibility of articles obtained in full-text. In accordance with current guidelines, inclusion of studies was determined by agreement between both reviewers [25].

\section{Assessment of quality}

The Effective Public Health Practice Project (EPHPP) Quality Assessment Tool for Quantitative Studies [26] was utilised to assess methodological quality of all included studies ${ }^{1}$. The EHPP was deemed more appropriate for the current review than the Cochrane Risk of Bias Tool [27, 28] because the EHPP was developed for use with all quantitative designs (not just RCTs) and has been found to have greater inter-rater agreement [29]. The EPHPP tool allows reviewers to derive a global rating of quality from the following components: selection bias, study design, confounders, blinding and withdrawals and drop-outs. Quality of included studies was independently assessed by two of the authors (EW \& KM), with disagreement resolved via discussion.

\footnotetext{
${ }^{1}$ See http://www.ephpp.ca/PDF/Quality\%20Assessment\%20 Tool_2010_2.pdf and http://www.ephpp.ca/PDF/QADictionary_ dec2009.pdf for Quality Assessment Tool.
} 


\section{Study synthesis}

In accordance with relevant guidelines, a narrative synthesis was deemed the most appropriate method of analysis due to substantial heterogeneity between included studies (e.g. design and outcome measures) which meant that meta-analysis was precluded [27, 30, 31]. A narrative synthesis should involve a preliminary synthesis of findings, an exploration of relationships in the data, an assessment of quality of the evidence and, if appropriate and feasible, the development of a theoretical model about the way in which an intervention works. Accordingly, quality appraisal, tabulation of the data, grouping by intervention type and study quality and analysis of variables influencing effectiveness were used as tools to facilitate the current narrative synthesis [30].

\section{RESULTS}

\section{Included studies}

After removal of duplicates, a total of 3,136 studies were extracted from online databases and other sources. Following primary screening, 3,117 studies were excluded as ineligible, with common reasons for exclusion comprising: not an intervention study, not an appropriate sample (condition or age), not a relevant outcome and not an empirical study. Thus, nineteen articles were retrieved as full-text for secondary screening, of which ten articles were included within the review (see Fig. 1 for study selection flow diagram).

\section{Study characteristics}

The vast majority of included studies ( 8 of the 10) were published in the last four years. Of the included studies, three were randomised controlled trials (RCT) [32-34], four were cohort analytic trials (CAT) [35-38] and three were cohort (pre-post) designs [39-41] (see Table 1 for intervention details and summary outcomes). Most studies were small, with the total number of participants ranging from eight $80(M=39.1)$. Participant demographics were varied. Samples consisted of between $32 \%$ and $74 \%$ males. Half of the interventions were for patients with ALS $[32,33,38,40,41]$. One study was for patients with post-polio syndrome [34], one study was for patients with mixed muscular dystrophies (e.g. facioscapuolohumeral muscular dystrophy, becker dystrophy and limb-girdle dystrophy etc.) [36] and the remaining three studies were for patients with mixed types of neuromuscular conditions (e.g. Charcot-Marie-Tooth disease, myasthenia gravis, post-polio syndrome and myotonic dystrophy etc.) (please see Table 1 for full list of conditions and sample sizes) [35, 37, 39]. Interventions were varied and included cognitive behavioural therapy [33, 34, 37], hypnosis [38, 40], dignity therapy [41], expressive disclosure[32], gratitude lists [35], comprehensive rehabilitation [36] and a psychoeducational fatigue management group [39]. The length of interventions ranged from one hour to 10 days, delivered over periods of between one week and 18 months. Six of the interventions were home-based [32, 35, 37, 38, 40, 41] and four were delivered in clinic settings [33, 34, 36, 39]. The majority of interventions were face to face $[33,34$, $36,38-41]$, with two delivered remotely via written instructions [32, 35], and one delivered online [37]. Outcomes pertaining to quality of life and well-being were measured in different ways across studies. Only four studies included a six month follow-up to investigate longer term effects of interventions [32-34, 38].

\section{Quality appraisal}

Using the EPHPP Quality Assessment Tool for Quantitative Studies [26], three RCTs were appraised as moderate in quality and the remainder were appraised as weak (see Table 2 for quality appraisal ratings). Amongst the three moderate studies, small sample sizes, insufficient blinding, selective outcome reporting and use of a non-active control were problematic. In one study, a strong design was hampered by slow recruitment and retention resulting in the trial being stopped prematurely [33]. Similar issues were identified in the seven studies appraised as weak. Of these studies, three used a non-equivalent control and three did not utilise any form of control. Most weak studies recruited participants from a single site or support group and thus are likely to be affected by selection bias. In many weak studies it was unclear whether co-intervention/contamination had occurred as this information was not clearly reported. Across all studies, blinding was particularly weak, with only two studies using blinded outcome assessors [33, 34]. Overall, fidelity to the intervention was not adequately assessed. In contrast, data collection (e.g. use of validated scales etc.) was generally strong.

\section{Study findings: Effectiveness of interventions}

Due to the heterogeneous nature of included studies, a meta-analysis was precluded. Instead, studies 


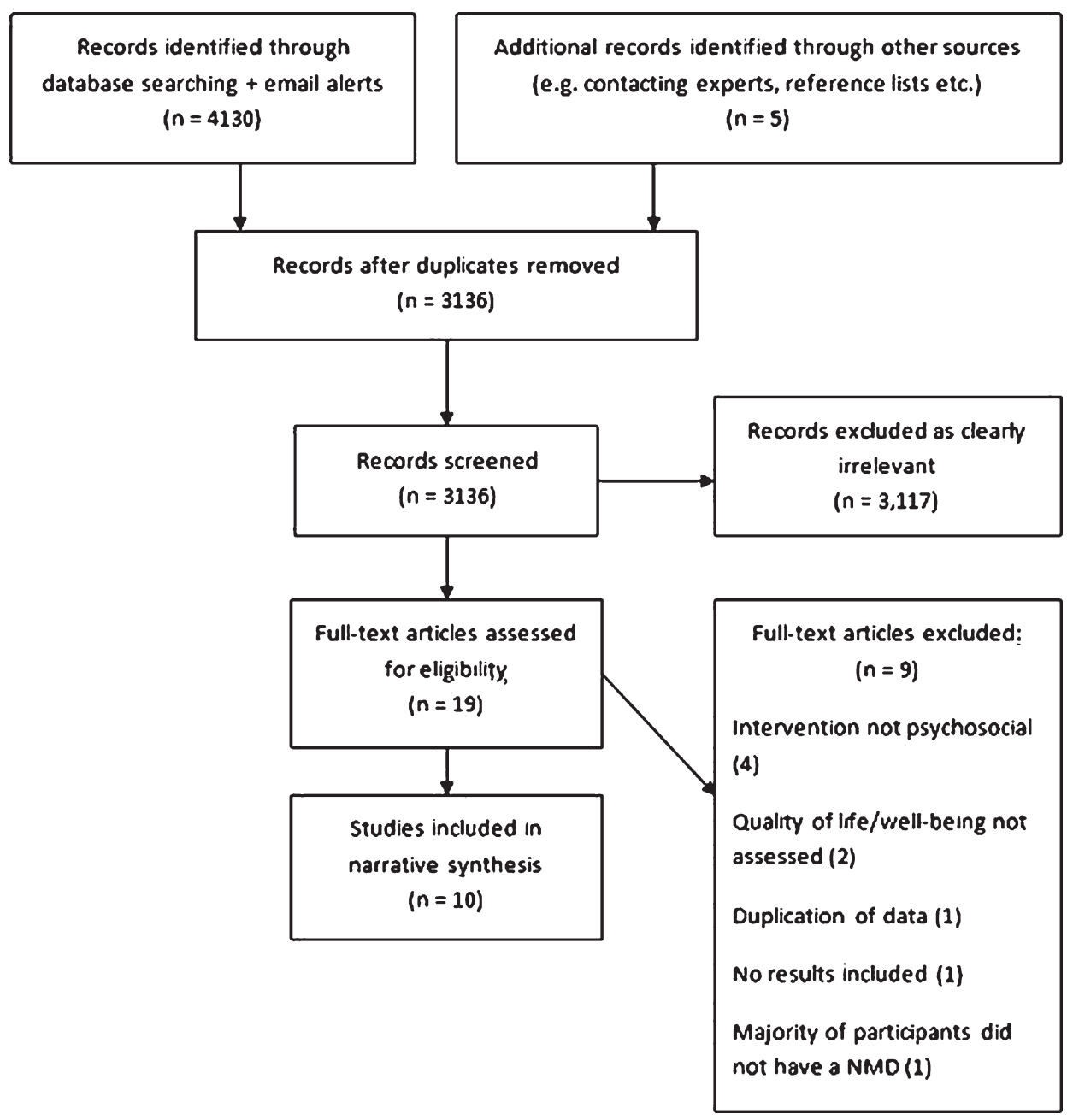

Fig. 1. Flow diagram of study selection process.

were grouped according to intervention type, outcome measurement and moderators of effectiveness as these groupings were deemed most pertinent to intervention effectiveness.

\section{Psychotherapy interventions}

Six studies investigated psychotherapy interventions, the most frequent of which was Cognitive Behavioural Therapy (CBT). One moderate quality RCT investigated CBT tailored to the management of fatigue in individuals with post-polio syndrome and self-reported severe fatigue [34]. The study found no difference in HRQoL between participants randomised to CBT and a usual care control group immediately post-intervention and at the three and six month follow-up. The authors reported a slightly greater occurrence of co-interventions (e.g. medication, assistive devices etc.) in the control group, which could have potentially confounded the results. However, neither the CBT or control group showed any improvement over time. The authors suggest that as post-polio syndrome has an early onset, acceptance of fatigue may develop over time, thus reducing the effectiveness of intervention [34].

In contrast, another moderate quality RCT investigated CBT based on a stress-coping model for patients with ALS and their partners [33]. The study found that, compared to a non-active control, intervention patients' mental quality of life deteriorated less over time between baseline and six month followup. However, this effect was only found in one of the two measures used. It should also be noted that the very small sample size in this study (total $n=15$ ) means that the statistical tests utilised were not 


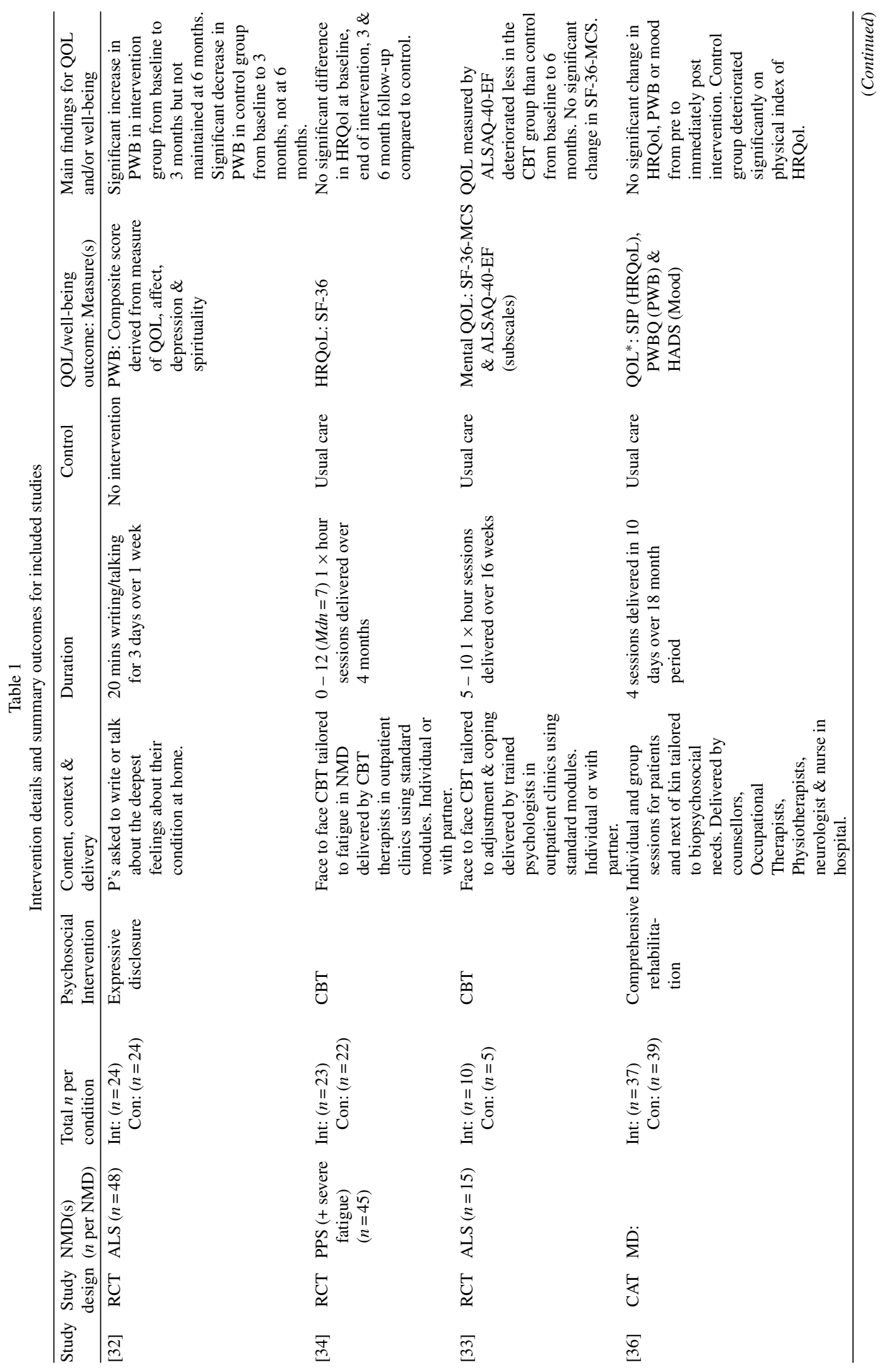




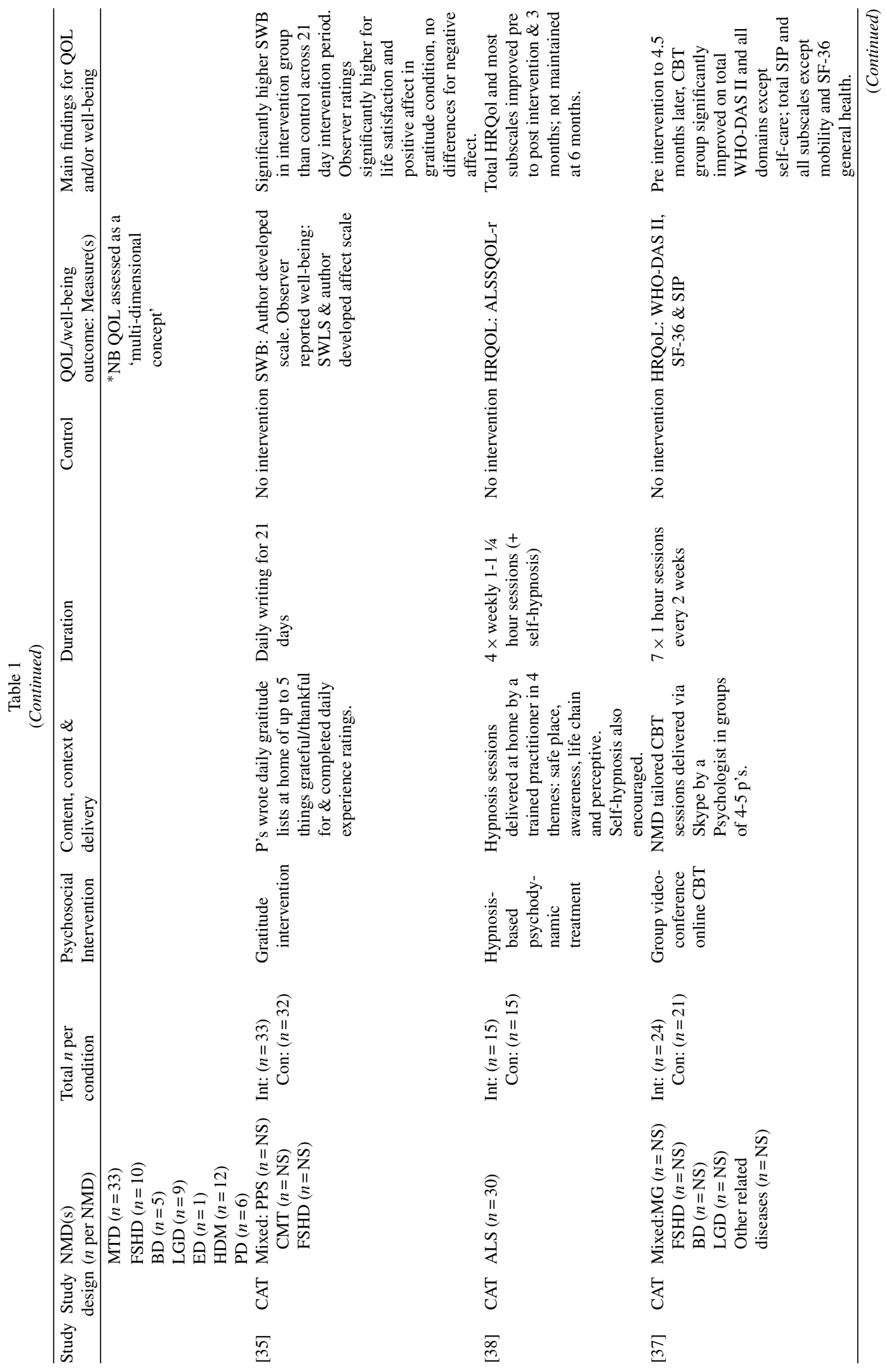




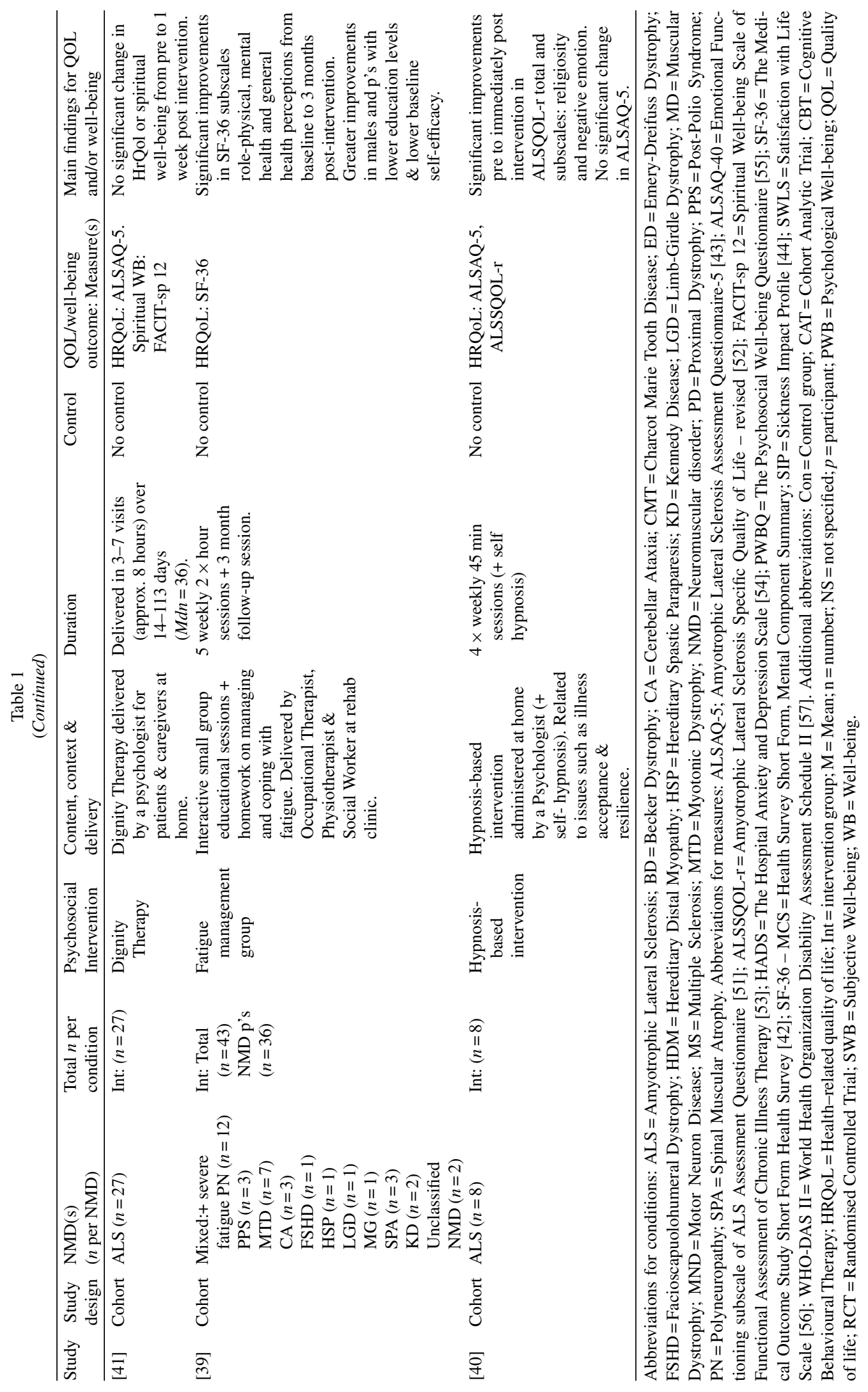


Table 2

Quality appraisal of all included studies

\begin{tabular}{|c|c|c|c|c|c|c|c|c|}
\hline$\overline{\text { Study }}$ & $\begin{array}{c}\text { Selection } \\
\text { Bias }\end{array}$ & $\begin{array}{c}\text { Study } \\
\text { Design }\end{array}$ & Confounds & Blinding & $\begin{array}{c}\text { Data } \\
\text { Collection }\end{array}$ & $\begin{array}{c}\text { Withdrawals/ } \\
\text { Dropouts }\end{array}$ & $\begin{array}{l}\text { Global } \\
\text { rating }\end{array}$ & $\begin{array}{l}\text { Additional } \\
\text { Notes }\end{array}$ \\
\hline [36] & Moderate & Moderate & Weak & Weak & Strong & Strong & Weak & $\begin{array}{l}\text { A small number of the control group } \\
\text { were also receiving rehabilitation. No } \\
\text { intervention protocol provided. } \\
\text { Quality and consistency of } \\
\text { intervention not measured. }\end{array}$ \\
\hline [41] & Weak & Moderate & N/A & Weak & Strong & Moderate & Weak & $\begin{array}{l}\text { No control group. Detailed protocol for } \\
\text { intervention provided and sessions } \\
\text { recorded but not rated for quality and } \\
\text { adherence. }\end{array}$ \\
\hline [32] & Moderate & Strong & Strong & Weak & Moderate & Strong & Moderate & $\begin{array}{l}\text { Selective outcome reporting. } \\
\text { Standardised instructions provided but } \\
\text { adherence to intervention not } \\
\text { measured. }\end{array}$ \\
\hline [39] & Weak & Moderate & N/A & Weak & Strong & Strong & Weak & $\begin{array}{l}\text { No control group. Content of } \\
\text { intervention described but no method } \\
\text { of checking quality and adherence. }\end{array}$ \\
\hline [35] & Weak & Weak & Weak & Weak & Weak & Weak & Weak & $\begin{array}{l}\text { Standardised instructions provided. } \\
\text { Manipulation check confirmed } \\
\text { gratitude condition elicited greater } \\
\text { expressions of gratitude than control. } \\
\text { Mean outcomes across intervention } \\
\text { period calculated for analyses. }\end{array}$ \\
\hline [38] & Weak & Moderate & Strong & Weak & Strong & Strong & Weak & $\begin{array}{l}\text { Small sample. Intervention protocol but } \\
\text { no method of checking quality and } \\
\text { adherence. } 5 \text { intervention participants } \\
\text { were also receiving } \\
\text { psychopharmacology }\end{array}$ \\
\hline [34] & Weak & Strong & Strong & Moderate & Strong & Moderate & Moderate & $\begin{array}{l}\text { Small sample. Standardised CBT } \\
\text { modules utilised and selected via } \\
\text { criteria. Quality and adherence not } \\
\text { assessed. Co-interventions received in } \\
\text { control and treatment groups. }\end{array}$ \\
\hline [37] & Weak & Moderate & Weak & Weak & Strong & Weak & Weak & $\begin{array}{l}\text { Participants assigned to control group if } \\
\text { they did not have access to a } \\
\text { computer/webcam. Detailed protocol } \\
\text { for intervention provided but no } \\
\text { method of checking quality and } \\
\text { adherence. Significant baseline } \\
\text { differences in quality of life not } \\
\text { controlled in analysis. }\end{array}$ \\
\hline [40] & Weak & Weak & N/A & Weak & Moderate & Strong & Weak & $\begin{array}{l}\text { No control group. Small sample size. } \\
\text { Intervention protocol but no method of } \\
\text { checking quality and adherence } \\
\text { described. }\end{array}$ \\
\hline [33] & Weak & Strong & Strong & Moderate & Strong & Strong & Moderate & $\begin{array}{l}\text { Small sample. Standardised CBT module } \\
\text { utilised but no method of checking } \\
\text { quality and adherence. Trial stopped } \\
\text { prematurely due to slow recruitment } \\
\text { and missing data. }\end{array}$ \\
\hline
\end{tabular}

Note: Global quality ratings were calculated from component ratings in accordance with criteria specified in the EPHPP tool. Global rating 'weak' = 2 + weak component ratings; global quality rating 'moderate' = 1 weak component rating; global quality rating 'strong' = no weak component ratings. See http://www.ephpp.ca/PDF/Quality\%20Assessment\%20Tool_2010_2.pdf.

adequately powered [33]. The contribution of these findings to the evidence base is therefore questionable.

Additionally, a CAT appraised as weak in quality investigated the efficacy of a CBT-based online intervention delivered via video conference [37]. The intervention was tailored to neuromuscular disorders and involved psychoeducation, managing emotional reactions, cognitive restructuring, problem solving and relaxation exercises for groups of patients with mixed neuromuscular disorders. Across the three different measures of HRQoL, the authors reported the 
intervention group significantly improved between baseline and follow-up (4 1/2 months later) on overall HRQoL and most subscales. It should be noted, however, that the intervention group had significantly lower levels of baseline HRQoL than the control group and at follow-up the two groups were comparable. As appropriate statistical analyses were not conducted to calculate the group $\times$ time interaction or control for baseline differences, the results may simply reflect regression to the mean in the intervention group.

Two linked, weak quality studies investigated the effect of a hypnosis intervention on quality of life and psychological well-being in patients with ALS. The first was a small pilot cohort study which assessed the impact of four sessions of Psychologist delivered hypnosis around issues such as illness acceptance, resilience and physical symptom control, in addition to self-hypnosis training [40]. In the absence of a control group, the authors reported significant improvements in one measure of HRQoL and subscales negative emotion and religiousness/spirituality. However, only eight participants took part in the study and therefore statistical tests were not adequately powered. Moreover, effects were not replicated in the second measure of HRQoL utilised in the study. Generalisability of the findings is therefore limited. This intervention was subsequently extended to a brief psychodynamic-based hypnosis intervention with four sessions on topics labelled safe place, awareness, life chain and perceptive [38]. Assessing the impact on HRQoL for 15 intervention participants and 15 matched controls, the authors reported a small effect of treatment on HRQoL immediately post intervention and at three months, with scores reverting towards baseline levels at six months. It should be noted however, that one third of the intervention group participants were receiving a low dose antidepressant or anxiolytic which could potentially confound the results.

The final weak cohort study assessed the impact of dignity therapy on HRQoL and spiritual wellbeing in patients with ALS and their caregivers [41]. Dignity therapy involves an in-depth interview with patients on their accomplishments in life and things they would like to pass on to loved ones which is later transcribed to create a permanent record for the individual and their family [41]. In a pre-post design the authors reported no significant impact on HRQoL or spiritual well-being one week post-intervention. Results are difficult to interpret due to the absence of a control. Moreover, the absence of an effect could potentially be influenced by the short follow-up time and brief measure used to assess HRQoL which may not be sensitive to change.

In summary, evidence for the effectiveness of psychotherapy interventions was mixed, with some types of psychotherapy found to be beneficial for quality of life and well-being (e.g. CBT and hypnosis) and some types found to be ineffective (e.g. CBT for fatigue and dignity therapy). Study quality was generally higher for CBT-related interventions than other forms of psychotherapy, however all studies had considerable methodological limitations.

\section{Private expressive communication}

Two studies examined the effects of private expressive communication interventions on well-being. One moderate quality RCT investigated expressive disclosure in patients with ALS [32]. Participants randomised to the intervention either wrote or audio recorded themselves speaking about their deepest thoughts in relation to their condition for three days in a one week period. Control participants completed outcome measures only. The authors reported a significant increase in psychological well-being from pre-post intervention in the expressive disclosure group, which was maintained at three months. In contrast, the control group experienced deterioration in psychological well-being from pre-post intervention which was also maintained at three months. Both groups reverted to near baseline levels at six months suggesting the effectiveness of the intervention was not maintained beyond three months. Furthermore, the use of a non-active control and selective outcome reporting (individual measures used to create a composite 'psychological wellbeing' score are not reported) limit the generalisability of findings.

An expressive communication intervention with a different emphasis was utilised by one CAT appraised as weak in quality [35]. In this gratitude intervention, participants with slowly progressive neuromuscular conditions (Charcot-Marie-Tooth disease, post-polio syndrome \& facioscapuolohumeral dystrophy) were asked to write down five things they were grateful for and complete experience ratings daily for 21 days. Control participants completed the experience ratings but were not asked to write gratitude lists. The study found the intervention group had significantly higher subjective well-being than the control group across the 21 day study period. It should be noted, however, that a three-item measure was developed by the author to assess subjective well-being and the reliability and 
validity of this measure are unknown. This study also assessed observer reports of well-being, with caregivers/spouses asked to complete measures of life satisfaction and positive and negative affect from the patient's perspective at the end of the intervention. The authors reported that life satisfaction and positive affect were rated significantly higher for intervention group patients compared with ratings for patients in the control group; no differences were reported for negative affect. The absence of any follow-up measures for this intervention beyond the 21 day study period mean it is not possible to establish whether effects were maintained.

In summary, there was some evidence for the effectiveness of expressive disclosure and gratitude lists in improving well-being, albeit in the short-term. As with psychotherapy interventions, the quality of evidence for private expressive communication interventions was limited.

\section{Group psychoeducation/Comprehensive rehabilitation}

Two weak quality studies assessed group-based psychosocial interventions delivered in clinic settings. One cohort study assessed an educational fatigue management intervention for patients with mixed slowly progressive neuromuscular conditions or multiple sclerosis [39]. Between baseline and three months post intervention, significant improvements were found in role-physical, mental health and general health perceptions subscales of the HRQoL measure SF-36 [42]. The absence of a control group makes the results difficult to interpret. The second study did utilise a control group (usual care) and found no significant change in HRQoL, psychological well-being or mood following a 10 day comprehensive rehabilitation programme for adults with muscular dystrophy [36]. It should be noted that a small number of the control group were also receiving rehabilitation which was not controlled for in the analyses.

In summary, whilst there was weak evidence for the effectiveness of group-based psychoeducation and the ineffectiveness of comprehensive rehabilitation, the absence of appropriate controls within studies inhibited meaningful interpretation of findings.

\section{Study findings: Assessment of quality of life and well-being}

Quality of life and well-being were defined and assessed differently across all included studies, thereby complicating interpretation of findings (see Table 1). In one study, psychological well-being was considered a multi-faceted concept incorporating HRQoL [32]. Conversely, in another study, quality of life was defined and assessed as a multi-faceted concept incorporating psychological well-being [36]. The majority of studies on ALS utilised one of several ALS specific measures of HRQoL. Both studies utilising the ALSAQ-5 [43] reported no significant effects, despite effects found in other similar measures in one of these studies [40,41]. This suggests that different measures of HRQoL may be measuring different constructs. Amongst other neuromuscular conditions, the SF-36 [42], and SIP [44] were most commonly used to assess generic HRQoL. The two studies utilising a specific validated measure of wellbeing (as opposed to a composite measure or author developed scale) did not report any beneficial effects $[36,41]$. Overall, outcome measurement was problematic and lacked a consistent approach across studies.

\section{Study findings: Factors influencing effectiveness}

Only two studies investigated factors which may have influenced effectiveness of the intervention on quality of life and/or well-being. Averill and colleagues [32] reported that higher ambivalence over expressing emotion was associated with greater increases in psychological-wellbeing three months after expressive disclosure. Boosman and colleagues [39] reported greater improvements in HRQoL following a fatigue management intervention in participants who were male, individuals with lower education levels and those with lower baseline selfefficacy.

A set of criteria were derived from interventions in the included studies. Subsequently, components of each intervention were extracted and compared to allow for the identification of variables influencing effectiveness (see Table 3). No clear findings emerged from this analysis. Further research examining potential moderating factors is, therefore, required.

\section{DISCUSSION}

\section{Key findings}

This systematic review sought to synthesise the available evidence for the effectiveness of psychosocial interventions on quality of life and wellbeing in adults with neuromuscular disorders. Furthermore, it 
Table 3

A comparison of intervention factors within included studies

\begin{tabular}{|c|c|c|c|c|c|c|c|c|c|}
\hline Study & $\begin{array}{c}\text { Theoretical } \\
\text { basis } \\
\text { specified }\end{array}$ & $\begin{array}{l}\text { Protocol/ } \\
\text { Manual }\end{array}$ & $\begin{array}{l}\text { Delivered by } \\
\text { Psychologist }\end{array}$ & $\begin{array}{c}\text { Provides } \\
\text { psycho-education }\end{array}$ & $\begin{array}{c}\text { Targets } \\
\text { emotional } \\
\text { expression }\end{array}$ & $\begin{array}{l}\text { Targets } \\
\text { relaxation }\end{array}$ & $\begin{array}{c}\text { Targets } \\
\text { cognitions }\end{array}$ & $\begin{array}{c}\text { Targets } \\
\text { behaviours }\end{array}$ & $\begin{array}{c}\text { Facilitates } \\
\text { social } \\
\text { support } \\
\end{array}$ \\
\hline$\overline{[34]}$ & $\checkmark$ & $\checkmark$ & $\checkmark$ & $\checkmark$ & $\mathrm{X}$ & $\mathrm{X}$ & $\checkmark$ & $\checkmark$ & $\checkmark$ \\
\hline [32] & $X$ & $\checkmark$ & $X$ & $\mathrm{X}$ & $\checkmark$ & $X$ & $\mathrm{X}$ & $\mathrm{X}$ & $X$ \\
\hline [33] & $\checkmark$ & $\checkmark$ & $\checkmark$ & $\checkmark$ & $\checkmark$ & $X$ & $\checkmark$ & $\checkmark$ & $\checkmark$ \\
\hline [36] & $X$ & $X$ & $X$ & $\checkmark$ & $X$ & $X$ & $X$ & $\checkmark$ & $\checkmark$ \\
\hline [35] & X & $\checkmark$ & $X$ & $\mathrm{X}$ & $\checkmark$ & $\mathrm{X}$ & $\mathrm{X}$ & $\mathrm{X}$ & $X$ \\
\hline [38] & $\checkmark$ & $\checkmark$ & $\checkmark$ & $\mathrm{X}$ & $\mathrm{X}$ & $\checkmark$ & $\mathrm{X}$ & $\mathrm{X}$ & $\mathrm{X}$ \\
\hline [37] & $\checkmark$ & $\checkmark$ & $\checkmark$ & $\checkmark$ & $\checkmark$ & $\checkmark$ & $\checkmark$ & $\checkmark$ & $\checkmark$ \\
\hline [41] & X & $\checkmark$ & $\checkmark$ & $\mathrm{X}$ & $\checkmark$ & X & X & X & X \\
\hline [39] & $X$ & $\checkmark$ & $X$ & & $X$ & $X$ & $X$ & $\checkmark$ & $\checkmark$ \\
\hline [40] & $X$ & $\checkmark$ & $\checkmark$ & $\mathrm{X}$ & $X$ & $\checkmark$ & $\mathrm{X}$ & $\mathrm{X}$ & $\mathrm{X}$ \\
\hline
\end{tabular}

$=$ No significant effects of intervention on quality of life and/or well-being. Note: Intervention factors were derived from variables within included studies.

sought to critically appraise the methodological quality of this evidence. Despite calls for research on psychosocial interventions in this patient group [14, 45], only a small number of studies have been conducted. Of the 10 included studies, seven reported a beneficial effect of psychosocial interventions across different neuromuscular disorders. Overall, there is mixed and contradictory evidence for the effectiveness of CBT in improving HRQoL and mental quality of life, with moderate evidence of its ineffectiveness in severely fatigued participants [33, 34, 37]. It should be noted that CBT is the only intervention where a beneficial effect was found to be maintained at six months post intervention [33]; however, this may reflect a paucity of longer-term follow-up data in other studies. In the short-term, there was moderate evidence for emotional disclosure improving psychological well-being [32], weak evidence for gratitude lists improving subjective well-being [35], and weak evidence for hypnosis $[38,40]$ and a fatigue psychoeducational group [39] improving HRQoL. In contrast, there was weak evidence to suggest that dignity therapy does not improve HRQoL and spiritual well-being [41] and that comprehensive rehabilitation does not improve global quality of life [36]. In summary, there is currently insufficient evidence to support the use of psychosocial interventions to improve quality of life and well-being in adults with neuromuscular disorders.

The majority of included studies were rated as weak in quality, with only three rated as moderate and none as strong. Across studies, factors relating to selection bias, study design and blinding were particularly problematic. The majority of studies recruited a small number of participants, often from a single source. Whilst this may reflect the rarity of neuro- muscular conditions and the challenges they pose to engagement in activities [24], the risk of biased sampling limits generalisability of findings. With respect to study design, none of the included studies utilised an active control and three studies included no form of control at all. Thus, positive findings could be due to non-specific treatment effects. Importantly, as many studies did not assess long-term outcomes of interventions, inferences regarding long-term effects cannot be made. In most studies there was no blinding, with participants and outcome assessors fully aware of the research question and group allocations. Furthermore, consistency of the intervention was rarely assessed beyond the development of treatment protocols. In the context of these numerous risks of bias, caution must be exercised in interpretation of findings.

Further challenges to useful synthesis within this systematic review arose from the widespread heterogeneity amongst included studies. In particular, contrasting definitions and assessment methods for quality of life and well-being impeded meaningful comparison across studies. It is possible that this is reflective of confusion surrounding these terms within the wider literature and the field of neuromuscular disease $[46,47]$. HRQoL was the most commonly assessed outcome, however many different measures were utilised. Across all outcomes, a diverse group of measures were employed including both disorder specific and generalised measures and both single measures and multiple/composite measures. Of further concern, some studies reported contradictory findings when different measures were utilised to assess the same construct within an individual study $[33,40]$, thereby providing evidence against the comparability of outcome measures. Thus, the 
extent to which quality of life and well-being were validly and reliably assessed is cause for concern.

Study heterogeneity also precluded the development of a theoretical model about the way in which the included interventions work. Identifying how, why and for whom interventions work are important considerations for understanding intervention effectiveness. [30]. However, the majority of studies did not specify a theoretical basis and findings relevant to moderators and mediators were limited. Moderate evidence suggests that greater benefits to psychological well-being were gained by individuals with ambivalence over emotional expression [32]. Additionally, limited evidence suggests that greater benefits to HRQoL were gained by men, individuals with lower levels of education, and those with poor self-efficacy [39]. At the intervention level, no component emerged as consistently associated with gains in quality of life or well-being. It is, therefore, not possible to conclude whether particular characteristics of participants and/or interventions are most likely to result in beneficial effects from psychosocial interventions.

\section{Implications for research}

Whilst still at an early stage, research in this field has developed rapidly in recent years, with all three randomised controlled trials published in the last two years. Although these developments are promising, methodological limitations are still endemic in the available literature. It is vital that research proceeds with a strong emphasis on study quality such that meaningful conclusions can be made. As the gold standard for intervention research, randomised controlled trials should be prioritised, with double-blind designs and multi-site recruitment employed where possible. Active controls should also be utilised to establish whether beneficial effects are due to the specific intervention or simply from non-specific effects associated with being part of an intervention. Caution should be exercised in the design of active controls however, since equivalent noneffective comparison interventions are challenging to develop in psychosocial research [48]. In developing interventions, researchers should ensure detailed protocols are utilised and treatment fidelity is assessed.

Given the degenerative nature of neuromuscular diseases [24], it is vital that long term outcomes of interventions are assessed. Additionally, to avoid compounding current confusion, researchers should clearly define outcomes of interest according to accepted understandings of quality of life and wellbeing. Validated, condition-specific measures of quality of life may be preferable to generalised measures [48]. Moreover, since HRQoL is currently the most frequently assessed outcome, greater emphasis on global quality of life and well-being would be useful to progress understanding of the potential impact of interventions in this patient population.

The design and evaluation of psychosocial interventions should be informed by relevant theory. Thus, future research could usefully include analysis of moderator and mediator variables to develop new and existing theoretical models, from which interventions could then be developed. For example, researchers could investigate which individuals are most likely to benefit from an intervention by assessing the impact of individual difference and demographic factors on outcomes. Additionally, researchers could assess the relative importance of potential mechanisms of change, such as acceptance and altered cognitions.

\section{Implications for clinical practice and policy}

There is currently insufficient evidence to conclusively recommend psychosocial interventions for adults with neuromuscular disorders. However, since no study reported adverse outcomes from intervention, it can be concluded that clinicians and researchers should proceed in the development and evaluation of appropriately designed psychosocial interventions. Whilst one of the three moderate quality studies reported no beneficial effect of CBT, it should be noted that this intervention focused only on management of fatigue, which the authors acknowledged was not an important goal for all participants [34]. Given the individual variation and broad impact of neuromuscular disorders in adulthood [24], it is imperative that psychosocial interventions are designed and delivered in a way that is patientcentred.

\section{Strengths and weaknesses of this review}

This review extends findings from the one previous review in this area [22] to synthesise current knowledge on the impact of psychosocial interventions on quality of life and well-being in adults with neuromuscular disorders. The broad and systematic search strategy is a particular strength of this review. The high proportion of irrelevant articles retrieved from the full search indicates the search strategy had low specificity. However, there is always a trade-off 
between sensitivity and specificity, with the potential for missing important studies when specificity is high [49]. Moreover, the results are in keeping with the previous similar review in which a small number of studies were included from a broad search [22]. The main limitation of the current review is the heterogeneity amongst included studies. Due to the various included study designs and outcomes measures, a meta-analysis could not be performed. Consequently, no statistical inferences regarding effectiveness can be made.

\section{CONCLUSION}

There is currently no strong evidence to determine whether psychosocial interventions improve quality of life and well-being in adults with neuromuscular disorders. Although some benefits to both quality of life and well-being have been identified from a number of psychosocial interventions, such benefits are almost exclusively short-term and subject to bias. The paucity of high quality research and confusion surrounding measurement of quality of life and well-being impede meaningful interpretation of findings. Further research with a strong emphasis on study quality, clearly defined outcomes and consideration of moderator variables is urgently required to progress current understanding and inform recommendations for practice. Given that the overall prevalence of neuromuscular disorders in adulthood is increasing [50], it is vital that the identification of effective, patient-centred interventions becomes a prominent research priority.

\section{ACKNOWLEDGMENTS}

We wish to thank the authors of all included studies for their helpful responses to questions. We would also like to thank the reviewers of this paper for their valuable feedback.

\section{CONFLICT OF INTEREST}

The authors have no conflict of interest to report.

\section{SUPPLEMENTARY MATERIAL}

The supplementary information is available in the electronic version of this article: http://dx.doi.org/ $10.3233 / J N D-160155$.

\section{REFERENCES}

[1] Piccininni M, Falsini C, Pizzi A. Quality of life in hereditary neuromuscular diseases. Acta Neurol Scand. 2004;109:1139. doi: 10.1046/j.1600-0404.2003.00185.x

[2] Shy ME, Rose MR. Charcot-Marie-Tooth disease impairs quality of life: Why? And how do we improve it? Neurology. 2005;65(6):790-1. doi: 10.1212/01.wnl.0000 181027

[3] World Health Organization QOL Group. The World Health Organization quality of life assessment (WHOQOL): Position paper from the World Health Organization. Soc Sci Med. 1995;41(10):1403-9. doi:10.1016/02779536(95)00112-K

[4] Testa M, Simonson D. Assessment of quality-of-life outcomes. N Engl J Med. 1996;334(13):835-40. doi: 10.1056/NEJM199603283341306

[5] Ozer S, Yildirim SA, Yilmaz O, Düger T, Yilmaz SA. Assessment of health-related quality of life, depression, and anxiety in slowly and rapidly progressive neuromuscular disorders. Neurosciences. 2010;15(3):177-83.

[6] Graham CD, Rose MR, Grunfeld EA, Kyle SD, Weinman J. A systematic review of quality of life in adults with muscle disease. J Neurol. 2011;258(9):1581-92. doi: 10.1007/s00415-011-6156-0

[7] Guérin E. Disentangling vitality, well-being, and quality of life: A conceptual examination emphasizing their similarities and differences with special application in the physical activity domain. J Phys Act Health. 2012;9(6):896-908.

[8] Upton D, Upton P. Psychology of wounds and wound care in clinical practice. Switzerland: Springer; 2015.

[9] Huber M, Knottnerus JA, Green L, Horst HVD, Jadad AR, Kromhout D, et al. How should we define health? Br Med J. 2011;343:d4163-d4163. doi: 10.1136/bmj.d4163

[10] Diener E. Guidelines for national indicators of subjective well-being and ill-being. Appl Res Qual Life. 2006;1(2):151-7. doi: 10.1007/s11482-006-9007-x

[11] Burns RA, Ma J. Examining the association between psychological wellbeing with daily and intra-individual variation in subjective wellbeing. Pers Individ Dif. 2015;82:34-9. doi: 10.1016/j.paid.2015.02.023

[12] McDonald E, Wiedenfeld SA, Hillel A, Carpenter CL, Walter RA. Survival in amyotrophic lateral sclerosis: The role of psychological factors. Arch Neurol. 1994;51(1):17-23. doi: 10.1001/archneur.1994.00540130027010

[13] Nierse CJ, Abma TA, Horemans AMC, van Engelen BGM. Research priorities of patients with neuromuscular disease. Disabil Rehabil. 2013;35(5):405-12. doi: $10.3109 / 09638288.2012 .694964$

[14] Graham CD, Simmons Z, Stuart SR, Rose MR. The potential of psychological interventions to improve quality of life and mood in muscle disorders. Muscle Nerve. 2015;52:131-6. doi:10.1002/mus. 24487

[15] Simmons Z, Bremer BA, Robbins RA, Walsh SM, Fischer S. Quality of life in ALS depends on factors other than strength and physical function. Neurol. 2000; 55(3):388-92. doi: http://dx.doi.org/10.1212/WNL.55.3.388.

[16] Roach AR, Averill AJ, Segerstrom SC, Kasarskis EJ. The dynamics of quality of life in ALS patients and caregivers. Ann Behav Med. 2009;37(2):197-206. doi: 10.1007/s12160-009-9092-9

[17] Graham CD, Weinman J, Sadjadi R, Chalder T, Petty $\mathrm{R}$, Hanna MG, et al. A multicentre postal survey investigating the contribution of illness perceptions, coping and optimism to quality of life and mood in adults with 
muscle disease. Clin Rehabil. 2014;28(5):508-19. doi: 10.1177/0269215513511340

[18] Ruddy R, House A. Psychosocial interventions for conversion disorder. Cochrane Database Syst Rev. 2005;(4). doi: 10.1002/14651858.CD005331.pub2

[19] Rehse B, Pukrop R. Effects of psychocsocial interventions on quality of life in adult cancer patients: Meta analysis of 37 published controlled outcome studies. Patient Educ Couns. 2003;50(2):179-86. doi:10.1016/S0738-3991(02) 00149-0

[20] Steed L, Cooke D, Newman S. A systematic review of psychosocial outcomes following education, self-management and psychological interventions in diabetes mellitus. Patient Educ Couns. 2003;51:5-15. doi: 10.1016/S07383991(02)00213-6

[21] Osborn RL, Demoncada AC, Feuerstein M. Psychosocial interventions for depression, anxiety, and quality of life in cancer survivors: Meta-analyses. Int $J$ Psychiatry Med. 2006;36(1):13-34. doi: 10.2190/EUFN-RV1KY3TR-FK0L

[22] Gould RL, Coulson MC, Brown RG, Goldstein LH, AlChalabi A, Howard RJ. Psychotherapy and pharmacotherapy interventions to reduce distress or improve well-being in people with amyotrophic lateral sclerosis: A systematic review. Amyotroph Lateral Scler Front Degener. 2015; 16(56):293-302. doi: 10.3109/21678421.2015.1062515

[23] Cup EH, Pieterse AJ, ten Broek-Pastoor JM, Munneke $M$, van Engelen BG, Hendricks HT, et al. Exercise therapy and other types of physical therapy for patients with neuromuscular diseases: A systematic Review. Arch Phys Med Rehabil. 2007;88(11):1452-64. doi: 10.1016/j.apmr.2007.07.024

[24] Hilton-Jones D, Freebody J, Stein J. Neuromuscular disorders in the adult: A practical manual. Oxford, UK: Oxford University Press;2011.

[25] Centre for Reviews and Dissemination. Systematic reviews: CRD's guidance for undertaking reviews in health care [Internet]. Centre for Reviews and Dissemination; 2009 [cited 2016 January 15]. Available from: http://www. york.ac.uk/crd/SysRev/!SSL!/WebHelp/SysRev3.htm

[26] Thomas H. Quality assessment tool for quantitative studies [Internet]. Effective Public Health Practice Project; 2003 [cited 2016 January 15]. Available from: http://www.ephpp. ca/PDF/Quality\%20Assessment\%20Tool_2010_2.pdf

[27] Higgins JPT, Green S (editors). Cochrane handbook for systematic reviews of interventions version 5.1.0 [updated March 2011][Internet]. The Cochrane Collaboration; 2011 [cited 2016 January 15]. Available from: www.cochranehandbook.org

[28] Higgins JPT, Altman DG, Gotzsche PC, Juni P, Moher D, Oxman AD, et al. The Cochrane Collaboration's tool for assessing risk of bias in randomised trials. Brit Med J. 2011;343:d5928-d5928.

[29] Armijo-Olivo S, Stiles CR, Hagen NA, Biondo PD, Cummings GG. Assessment of study quality for systematic reviews: A comparison of the Cochrane Collaboration Risk of Bias Tool and the Effective Public Health Practice Project Quality Assessment Tool: Methodological research. J Eval Clin Pract. 2012;18(1):12-8. doi: 10.1111/j.13652753.2010.01516.x

[30] Popay J, Roberts H, Sowden A, Petticrew M, Arai L, Rodgers $\mathrm{M}$, et al. Guidance on the conduct of narrative synthesis in systematic reviews: A product of the ESRC methods programme. Lancaster: ESRC Method Programme; 2006.
[31] Ryan R. Heterogeneity and subgroup analyses in cochrane consumers and communication review group reviews: Planning the analysis at protocol stage [online]. Cochrane Consumers and Communication Review Group; 2014 [cited 2016 January 15]. Available from: http://cccrg.cochrane.org

[32] Averill AJ, Kasarskis EJ, Segerstrom SC. Expressive disclosure to improve well-being in patients with amyotrophic lateral sclerosis: A randomised, controlled trial. Psychol Health. 2013;28(6):701-13. doi: $10.1080 / 08870446.2012 .754891$

[33] van Groenestijn AC, Schröder CD, Visser-Meily JMA, Reenen ETK-V, Veldink JH, van den Berg LH. Cognitive behavioural therapy and quality of life in psychologically distressed patients with amyotrophic lateral sclerosis and their caregivers: Results of a prematurely stopped randomized controlled trial. Amyotroph Lateral Scler Front Degener. 2015;16(5-6):309-15. doi: 10.3109/21678421.2015.1038276

[34] Koopman FS, Voorn EL, Beelen A, Bleijenberg G, de Visser M, Brehm MA, et al. No reduction of severe fatigue in patients with postpolio syndrome by exercise therapy or cognitive behavioral therapy: Results of an RCT. Neurorehabil Neural Repair. 2016;30(5):402-10. doi: $10.1177 / 1545968315600271$

[35] Emmons RA, McCullough ME. Counting blessings versus burdens: An experimental investigation of gratitude and subjective well-being in daily life. J Pers Soc Psychol. 2003;84(2):377-89. doi: 10.1037/0022-3514.84.2. 377

[36] Ahlström G, Lindvall B, Wenneberg S, Gunnarsson LG. A comprehensive rehabilitation programme tailored to the needs of adults with muscular dystrophy. Clin Rehabil. 2006;20:132-41. doi: 10.1191/0269215506cr898oa

[37] Martínez O, Jometón A, Pérez M, Lázaro E, Amayra I, López-Paz JF, et al. Effectiveness of teleassistance at improving quality of life in people with neuromuscular diseases. Span J Psychol. 2014;17:1-9. doi: 10.1017/sjp.2014.89

[38] Kleinbub JR, Palmieri A, Broggio A, Pagnini F, Benelli E, Sambin M, et al. Hypnosis-based psychodynamic treatment in ALS: A longitudinal study on patients and their caregivers. Front Psychol. 2015;6:1-14. doi: 10.3389/fpsyg.2015.00822

[39] Boosman H, Visser-Meily JMA, Meijer J-WG, Elsinga A, Post MWM. Evaluation of change in fatigue, self-efficacy and health-related quality of life, after a group educational intervention programme for persons with neuromuscular diseases or multiple sclerosis: A pilot study. Disabil Rehabil. 2011;33(8):690-6. doi: 10.3109/09638288.2010.510176

[40] Palmieri A, Kleinbub JR, Calvo V, Sorarù G, Grasso I, Messina I, et al. Efficacy of hypnosis-based treatment in amyotrophic lateral sclerosis: A pilot study. Front Psychol. 2012;3:1-6. doi: 10.3389/fpsyg.2012.00465

[41] Aoun SM, Chochinov HM, Kristjanson LJ. Dignity therapy for people with motor neuron disease and their family caregivers: A feasibility study. J Palliat Med. 2015;18(1):31-7. doi: 10.1089/jpm.2014.0213

[42] Ware JE, Sherbourne CD. The MOS 36-Item Short-Form Health Survey (SF-36). Conceptual Framework and Item Selection. Med Care. 2012;30(6):473-83.

[43] Jenkinson C, Fitzpatrick R, Swash M, Jones G. Comparison of the 40-item Amyotrophic Lateral Sclerosis Assessment Questionnaire (ALSAQ-40) with a short-form five-item version (ALSAQ-5) in a longitudinal survey. Clin Rehabil. 2007;21:266-72. 
[44] Bergner M, Bobbitt RA, Kressel S, Pollard WE, Gilson BS, Morris JR. The sickness impact profile: Conceptual formulation and methodology for the development of a health status measure. Int J Heal Serv. 1976;6(3):393-415.

[45] Pagnini F, Simmons Z, Corbo M, Molinari E. Amyotrophic lateral sclerosis: Time for research on psychological intervention? Amyotroph Lateral Scler. 2012;13(5):416-7. doi: 10.3109/17482968.2011.653572

[46] Camfield L, Skevington SM. On subjective well-being and quality of life. J Health Psychol. 2008;13(6):764-75. doi: 10.1177/1359105308093860

[47] Burns TM, Graham CD, Rose MR, Simmons Z. Quality of life and measures of quality of life in patients with neuromuscular disorders. Muscle Nerve. 2012;46(1):9-25. doi: 10.1002/mus. 23245

[48] Stice E, Burton E, Bearman SK, Rohde P. Randomized trial of a brief depression prevention program: An elusive search for a psychosocial placebo control condition. Behav Res Ther. 2007;45(5):863-76. doi: 10.1016/j.brat.2006.08.008

[49] Harden A, Peersman G, Oliver S, Oakley A. Identifying primary research on electronic databases to inform decision-making in health promotion: The case of sexual health promotion. Health Educ J. 1999;58:290-301. doi: $10.1177 / 001789699905800310$

[50] Deenen JCW, Horlings CGC, Verschuuren JJGM. The epidemiology of neuromuscular disorders: A comprehensive overview of the literature. J Neuromuscul Dis. 2015;2:7385. doi: $10.3233 / J N D-140045$
[51] Jenkinson C, Fitzpatrick R, Brennan C, Bromberg M, Swash M. Development and validation of a short measure of health status for individuals with amyotrophic lateral sclerosis/motor neurone disease: The ALSAQ-40. J Neurol. 1999;246:16-21.

[52] Felgoise SH, Walsh SM, Stephens HE, Brothers A, Simmons Z. The ALS specific quality of liferevised (ALSSQOL-R) user's guide [Internet]. 2011 [cited 2016 January 15]. Available from: http://www. pennstatehershey.org/c/document_library/get_file?uuid=b9 de0a6a-9c1d-4f77-bdf0-5c6c846e018e\&groupId=22147

[53] Peterman AH, Fitchett G, Brady MJ, Hernandez L, Cella D. Measuring spiritual well- in people with cancer: The functional assessment of chronic illness therapyspiritual well-being scale (FACIT-Sp). Ann Behav Med. 2002;24(17):49-58. doi: 10.1207/S15324796ABM2401_06

[54] Zigmond AS, Snaith RP. The hospital anxiety and depression scale. Acta Psychiatr Scand. 1983;67:361-70.

[55] Kaasa S, Mastekaasa A, Naess S. Quality of life of lung cancer patients in a randomized clinical trial evaluated by a psychosocial well-being questionnaire. Acta Oncol. 1988;27:335-42.

[56] Diener E, Emmons RA, Larsen RJ, Griffin S. The Satisfaction With Life Scale. J Pers Assess. 1985;49(1):71-5.

[57] World Health Organization. World Health Organization disabilty assessment schedule: WHODAS II. Geneva: World Health Organization; 2000. 